
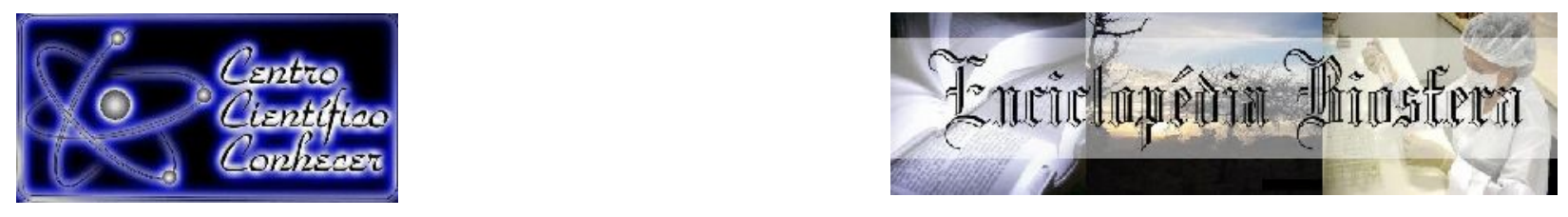

\title{
METODOLOGIA PARA ELABORAÇÃO DE UM SISTEMA FUZZY NA OBTENÇÃO DO ÍNDICE DE CONICIDADE EM ADULTOS
}

Giovana Xavier Pinto ${ }^{1}$; Camila Pires Cremasco²; Jhonatan Cabrera Piazentin ${ }^{3}$; Juliana Edwiges Martinez Spada"; Luís Roberto Almeida Gabriel Filho ${ }^{5}$.

${ }^{1}$ Graduanda em Fisioterapia, Faculdade da Alta Paulista (FADAP/FAP), Tupã, Brasil, giovanax07@hotmail.com

${ }^{2}$ Livre-Docente em Matemática Aplicada, Universidade Estadual Paulista (UNESP), Faculdade de Ciências e Engenharia, Tupã, Brasil.

${ }^{3}$ Mestrando, Programa de Pós-Graduação em Irrigação e Drenagem, Universidade Estadual Paulista (UNESP), Faculdade de Ciências Agronômicas, Botucatu, Brasil.

4. Mestre, docente do curso de Fisioterapia, Faculdade da Alta Paulista (FADAP/FAP), Tupã (SP). Doutoranda, Programa de Pós-Graduação em Avaliação e Intervenção em Fisioterapia, Universidade Estadual Paulista (UNESP), Faculdade de Ciências e Tecnologia, Presidente Prudente, Brasil.

${ }^{5}$ Livre-Docente em Matemática Aplicada e Computacional, Universidade Estadual Paulista (UNESP), Faculdade de Ciências e Engenharia, Tupã, Brasil.

\section{Recebido em: 06/04/2019 - Aprovado em: 10/06/2019 - Publicado em: 30/06/2019 DOI: 10.18677/EnciBio_2019A184}

Sabe-se que o excesso de massa corporal, sobretudo na região abdominal contribui para uma variedade de consequências à saúde dentre estas: dislipidemias e doenças cardiovasculares, desse modo o índice de conicidade (IC) pode ser uma ótima ferramenta para representar a obesidade abdominal em adultos. A fim de auxiliar os pesquisadores na área da saúde no processo de tomada de decisão, a lógica fuzzy vem sendo utilizada de modo inovador, por ser uma das ferramentas de inteligência artificial. Entretanto, é escasso seu uso em indicadores antropométricos. Assim, o presente trabalho teve por objetivo a elaboração de uma metodologia que será utilizada como ferramenta computacional baseada em lógica fuzzy para obtenção e avaliação do IC. A metodologia utilizada foi baseada na pesquisa quantitativa analítica e descritiva com delineamento transversal. Para a elaboração do modelo matemático apenas de maneira teórica, foram definidas as variáveis de entrada e saída ao índice a ser estudado, para as variáveis de entrada foram utilizadas: circunferência da cintura $(\mathrm{m})$, massa corporal $(\mathrm{kg})$ e altura $(\mathrm{m})$, e como a variável de saída o IC. Para a implementação computacional, utilizou-se o software Matlab®7.0 (The MathWorks Inc.) por meio do pacote Fuzzy Logic Toolbx. Concluise que o uso da lógica fuzzy pode contribuir significativamente na análise da obesidade abdominal auxiliando profissionais e estudantes que queiram fazer o uso do IC em suas avaliações antropométricas. Além disso, este modelo é capaz de imitar parte do raciocínio humano, e foi baseado em lógica fuzzy.

PALAVRAS-CHAVE: Circunferência da Cintura, Inteligência Artificial, Obesidade Abdominal. 


\title{
METHODOLOGY FOR THE DEVELOPMENT OF A FUZZY SYSTEM IN THE OBTAINMENT OF THE CONICET INDEX IN ADULTS
}

\begin{abstract}
It is known that excess weight, especially in the abdominal region contributes to a variety of health consequences among them: dyslipidemias and cardiovascular diseases, thus the conicity index $(\mathrm{Cl})$ can be a great tool to represent abdominal obesity in adults. In order to assist health researchers in the decision-making process, fuzzy Logic has been used in an innovative way, since it is one of the artificial intelligence tools. However, its use in anthropometric indicators is scarce. Thus, the objective of this work was the elaboration of a methodology that will be used as a computational tool based on fuzzy logic to obtain and evaluate the CI. The methodology used was based on quantitative analytical and descriptive research with a cross - sectional design. The variables of entry and exit to the index to be studied were defined for the input variables: waist circumference $(\mathrm{m})$, body weight $(\mathrm{kg})$ and height $(\mathrm{m})$, and as the output variable the IC. For its computational implementation, Matlab®7.0 software (The MathWorks Inc.) was used through the Fuzzy Logic Toolbx package. It is concluded that the use of fuzzy logic can contribute significantly to the analysis of abdominal obesity, helping professionals and students who want to use IC in their anthropometric evaluations. In addition, this model is able to mimic part of human reasoning, and was based on fuzzy logic.
\end{abstract}

KEYWORDS: Waist Circumference, Artificial Intelligence, Abdominal Obesity.

\section{INTRODUÇÃO}

A obesidade é um alarmante problema na saúde pública mundial, tanto nos países desenvolvidos quanto naqueles em desenvolvimento, e tem se mostrado em crescimento exponencial nas últimas décadas, triplicando desde 1975 (WHO, 2018). Segundo um levantamento de dados em 2016, mais de 1,9 bilhão de adultos com idade superior a 18 anos, apresentaram excesso de massa corporal, e mais de 650 milhões eram obesos (WHO, 2018). Nesse contexto, é importante ressaltar que o excesso de gordura corporal compromete e pode ocasionar danos à saúde como: doenças cardiovasculares, que são consideradas as principais causas de mortes no Brasil, câncer, alterações metabólicas, dificuldades respiratórias, bem como danos ao aparelho locomotor (AZEVEDO et al., 2014; PERRONE FILARDI et al., 2015; MALTA et al., 2017).

Há um entendimento na literatura de que o excesso de massa corporal, sobretudo na região abdominal contribui significativamente para uma variedade de consequências a saúde tais como: dislipidemias, hipertensão arterial, resistência à insulina e diabetes (TONSTAD; HJERMANN et al., 2003; OLIVEIRA et al., 2010). Diante desse contexto diferentes ferramentas antropométricas vêm sendo utilizadas, como a medida da circunferência da cintura, que atualmente é o método mais encontrado em estudos científicos para estabelecer a adiposidade abdominal (HAUN et al., 2009) sendo esta aplicada no cálculo IC proposto por Rodolfo Valdez em 1991.

O IC é uma ferramenta antropométrica, que tem como intuito indicar a obesidade abdominal do indivíduo. Este índice é considerado como um bom índice para avaliar os fatores de riscos cardiovasculares em indivíduos adultos (PITANGA; LESSA, 2007; MORAIS et al., 2018). O mesmo possui uma faixa teórica que para adultos é de 1,00 a 1,73 (valor adimensional). Valores próximos a 1,00 representam baixo risco de desenvolvimento de doenças cardiovasculares, entretanto quando 0 
indivíduo estiver próximo do valor 1,73 há elevado indicativo de risco cardiovascular (VALDEZ et al.,1993). No entanto, há limitações para seu uso, justamente pelo fato de serem escassos os pontos de corte para definir o risco de doenças cardiovasculares e também por exigir conhecimentos matemáticos para a realização do cálculo.

Neste contexto, com o crescente avanço computacional e matemático, sistemas de inteligência artificial vem contribuindo e tendo um grande destaque para os pesquisadores da área da saúde, por simularem o raciocínio humano, dessa forma contribuindo com as interpretações no processo de tomada de decisão para a propensão ou não ao desenvolvimento de doença com base nos conhecimentos passados ao sistema pelo avaliador. Utilizando-se redes neurais, foi estabelecido um primeiro resultado neste sentido em Pinto et al., (2018).

O objetivo deste presente trabalho é estabelecer as etapas iniciais da contrução agora de um modelo baseado em regras fuzzy para indicadores antropométricos. O presente trabalho teve como objetivo a elaboração de uma metodologia para desenvolvimento de uma ferramenta computacional baseada em lógica fuzzy, por meio do software Matlab®7.0 (The MathWorks) para obtenção e avaliação do IC, cujo modelo neste trabalho foi denominado IC Fuzzy.

\section{MATERIAIS E MÉTODOS}

\section{Método}

Tratou-se de uma pesquisa quantitativa analítica e descritiva com delineamento transversal.

\section{Elaboração do Modelo}

O estudo foi realizado entre janeiro e março de 2019, no Laboratório de Matemática Aplicada e Computacional (LabMAC), pertinente a Faculdade de Ciências e Engenharia FCE-UNESP, campus de Tupã-SP. Inicialmente, elaborou-se um modelo matemático sem regras, baseado em dados não reais. Após isso definiuse as variáveis de entrada, e as variáveis de saída em relação ao índice estudado conforme a Equação 1 proposta por Valdez (1991):

$$
I C=\frac{\text { circunferência da cintura }}{0,109 \sqrt{\frac{\text { massa corporal }}{\text { altura }}}}
$$

Para as variáveis de entrada do modelo utilizou-se circunferência da cintura $(\mathrm{m})$, massa corporal $(\mathrm{kg})$ e altura $(\mathrm{m})$ e como variável de saída o IC Fuzzy, e a constante 0.109 resultante da raiz da razão entre $4 \pi$ advindo do perímetro do círculo de um cilindro e a densidade média do ser humano adulto de $1,050 \mathrm{~kg} / \mathrm{m}^{3}$ (VALDEZ, 1991). 


\section{Desenvolvimento do estudo e implementação computacional}

O modelo baseado em sistema fuzzy para avaliação e obtenção do IC em adultos, utilizou-se como variáveis de entrada: circunferência da cintura $(\mathrm{m})$, massa corporal $(\mathrm{Kg})$ e altura $(\mathrm{m})$ a fim de determinar a variável de saída desejada, ou seja, o IC denominado como IC Fuzzy (Figura 1).

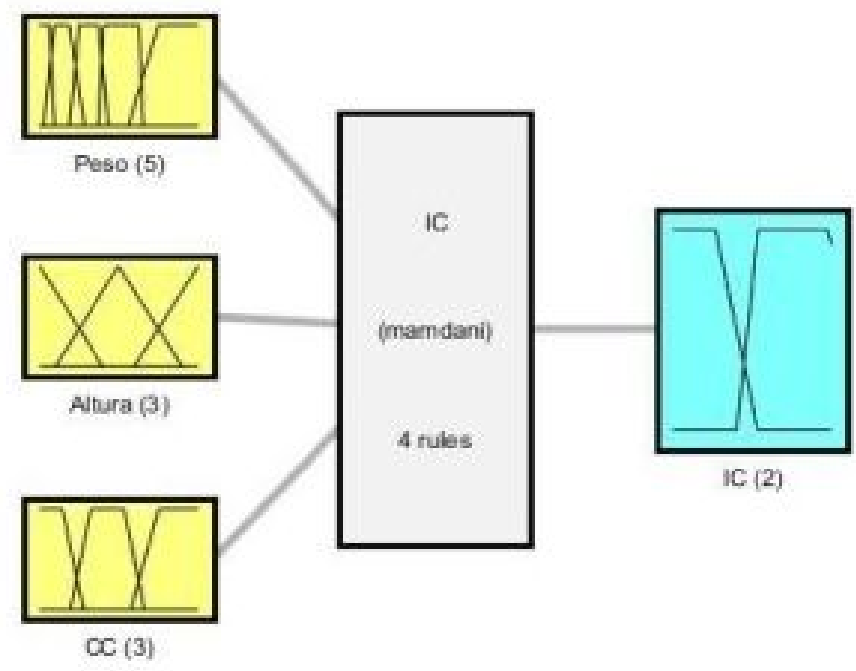

FIGURA 1. Modelo para avaliação do IC com 3 variáveis de entrada massa corporal, altura e circunferência da cintura, uma variável de saída índice de conicidade, IC Fuzzy. Fonte: (Elaborada pelos autores)

Todo processo de elaboração do modelo foi embasado na fórmula do IC (VALDEZ 1991). Para a implementação computacional, utilizou-se o software Matlab 7.0 (The MathWorks Inc) por meio do pacote Fuzzy Logic Toolbox, cuja Faculdade de Ciências e Engenharia FCE-UNESP, campus de Tupã-SP possui licença.

Foram gerados conjuntos fuzzy para cada variável do modelo. Tais conjuntos foram descritos por suas respectivas funções de pertinência, o que permite assim relacionar cada valor de cada variável a partir de graus de pertinência específicos à no máximo dois conjuntos fuzzy distintos. Além disto, tal construção dá suporte a criação de um sistema baseado em regras fuzzy propriamente dita, o que poderá ser feito em trabalhos futuros com a efetiva coleta de medidas antropométricas. Sendo assim, tal definição de conjuntos fuzzy torna-se primordial para a consolidação de tal sistema.

Destaca-se que as definições dos delimitadores das funções de pertinência das seguintes variáveis: massa corporal, altura, circunferência da cintura foram feitas apenas de maneira teórica, ou seja, o modelo não foi testado com dados reais, dessa forma foram colocados dados aleatórios, embora para o IC os delimitadores da função de pertinência foram feitos com base no intervalo sugerido por Valdez et al. (1993) que compreende em um intervalo de 1,0 a 1,73. Buscou-se definir, para cada tipo de variável, ou funções de pertinência triangulares ou trapezoidais. As funções do tipo triangulares da forma descrita a seguir: 


$$
\mu_{A}(x)= \begin{cases}0, & \text { se } x \leq a \\ \frac{x-a}{b-a}, & \text { se } a<x \leq b \\ \frac{x-c}{b-c}, & \text { se } b<x \leq c \\ 0, & \text { se } x>c\end{cases}
$$

para $a<b<c$.

Já as funções de pertinência do tipo trapezoidais, são descritas da seguinte forma:

$$
\mu_{A}(x)= \begin{cases}0, & \text { se } x \leq a \\ \frac{x-a}{b-a}, & \text { se } a<x \leq b \\ 1, & \text { se } b<x \leq c \\ \frac{x-c}{b-c}, & \text { se } c<x \leq d \\ 0, & \text { se } x>d\end{cases}
$$

Para cada variável do modelo definiu-se um conjunto fuzzy sendo assim para a variável massa corporal, definiram-se cinco conjuntos fuzzy trapezoidais, e as funções de pertinências foram denominadas como muito baixo (MB), baixo $(B)$, adequado (AD), alto (A), muito alto (MA), uma vez que buscou-se definir certos intervalos com pertinência somente para conjunto fuzzy. Para a variável altura definiram-se três conjuntos fuzzy triangulares, baixa $(B)$, média $(M)$ e alta $(A)$ e para a circunferência da cintura definiram-se três conjuntos fuzzy trapezoidais, denominada baixa $(B)$, média $(\mathrm{M})$ e alta $(\mathrm{A})$. Para a variável de saída, IC fuzzy, elaborou-se com dois conjuntos fuzzy trapezoidais, denominando estes conjuntos como risco baixo $(\mathrm{RB})$ e risco alto $(\mathrm{RA})$.

\section{RESULTADOS E DISCUSSÃO}

Desenvolveu-se um modelo em três etapas, sendo a primeira chamada de fuzzificação etapa na qual os dados são apresentados ao modelo, ou seja, são inseridas as variáveis de entrada; segunda etapa de inferência responsável pelo processamento dos dados; e terceira de defuzzificação processo pelo qual gera uma resposta de saída, neste caso IC Fuzzy.

Ressalta-se que o presente estudo preocupou-se em elaborar as funções de pertinência do modelo teórico. O sistema foi elaborado e testado com dados não reais, e sim aleatórios. As etapas do modelo justificam o fato da Lógica fuzzy simular o raciocínio humano e pertencer a área de inteligência artificial, justamente por ser comparada ao sistema nervoso (SN) o qual é constituído por milhões de células nervosas, e desempenha três funções fundamentais que se assemelham as etapas da Lógica fuzzy: captação de informações, através dos receptores sensitivos; processa e interpreta essas informações processo este chamado de integração; indica uma resposta de saída ativando os órgãos efetores em prol do organismo (MARIEB et al., 2014). 
De acordo com o Quadro 1, em relação a variável massa corporal, foram definidas cinco funções de pertinências denominadas muito baixo $(M B)$, baixo $(B)$, adequado $(A D)$, alto $(A)$, muito alto $(M A)$, cujos delimitadores para esta função de pertinência foram determinados como tipo trapezoidal. Este procedimento foi utilizado semelhantemente por Pereira et al., (2008); Cremasco et al. (2010), Gabriel Filho et al. (2015), e Putti et al. (2014, 2017).

QUADRO 1. Definição das funções de pertinência da variável de entrada massa corporal.

\begin{tabular}{|c|c|c|}
\hline Conjuntos Fuzzy & Tipo & Delimitadores \\
\hline $\mathrm{MB}$ & Trapezoidal & {$\left[\begin{array}{llll}-20 & 0 & 20 & 40\end{array}\right]$} \\
\hline $\mathrm{B}$ & Trapezoidal & {$\left[\begin{array}{llll}21 & 35 & 45 & 65\end{array}\right]$} \\
\hline AD & Trapezoidal & {$\left[\begin{array}{llllll}45 & 60 & 80 & 90\end{array}\right]$} \\
\hline A & Trapezoidal & {$\left[\begin{array}{lllll}80 & 85 & 120 & 125\end{array}\right]$} \\
\hline MA & Trapezoidal & {$\left[\begin{array}{lllll}110 & 140 & 185 & 200\end{array}\right]$} \\
\hline
\end{tabular}

Para a variável altura, foram definidas três funções de pertinência denominados baixa $(B)$, média $(M)$ e alta $(A)$, cujos delimitadores para esta função de pertinência foram determinados como tipo triangular (Quadro 2).

QUADRO 2. Definição das funções de pertinência da variável de entrada altura.

\begin{tabular}{ccc}
\hline Conjuntos Fuzzy & Tipo & Delimitadores \\
\hline$B$ & Triangular & {$[-0,400,4]$} \\
\hline$M$ & Triangular & {$[0,10,50,9]$} \\
\hline$A$ & Triangular & {$[0,611,6]$} \\
\hline
\end{tabular}

Para a variável circunferência da cintura, foram definidas três funções de pertinência denominadas como baixa $(B)$, média $(M)$ e alta $(A)$, cujos delimitadores para esta função de pertinência foram determinados como tipo trapezoidal (Quadro 3).

QUADRO 3. Definição das funções de pertinência da variável de entrada circunferência da cintura

\begin{tabular}{|c|c|c|}
\hline Conjuntos Fuzzy & Tipo & Delimitadores \\
\hline $\mathrm{B}$ & Trapezoidal & {$\left[\begin{array}{lllll}-50 & 50 & 60 & 70\end{array}\right.$} \\
\hline M & Trapezoidal & {$\left[\begin{array}{llll}65 & 70 & 90 & 95\end{array}\right]$} \\
\hline$A$ & Trapezoidal & {$\left[\begin{array}{lllll}90 & 100 & 125 & 150\end{array}\right]$} \\
\hline
\end{tabular}

As funções de pertinência das variáveis de entrada estão representadas na Figura 2 sendo (a) massa corporal, (b) altura e (c) circunferência da cintura. 


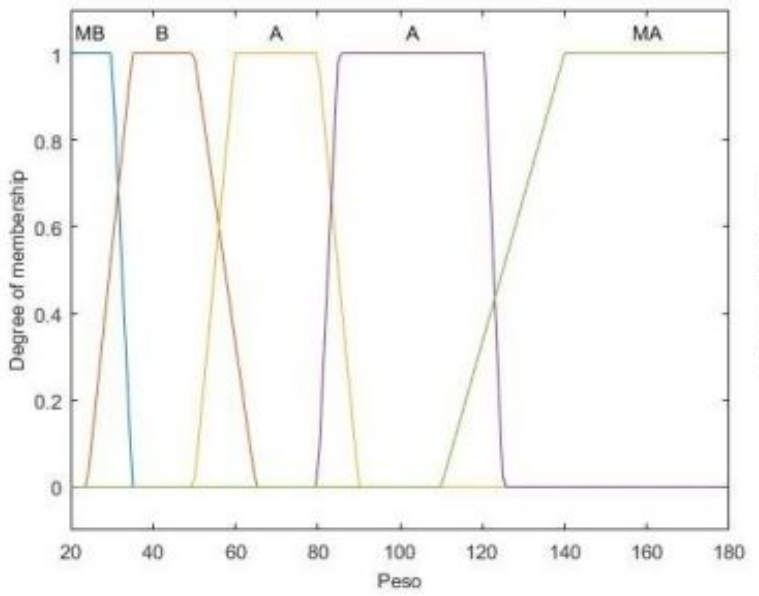

(a)

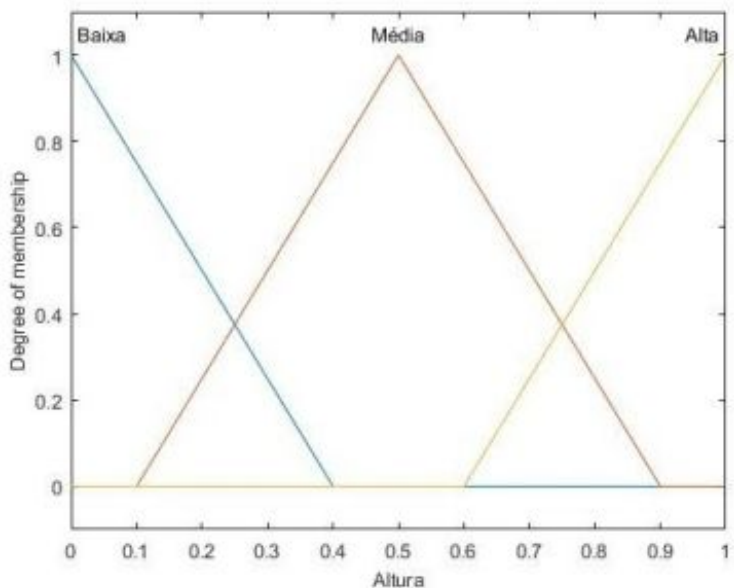

(b)

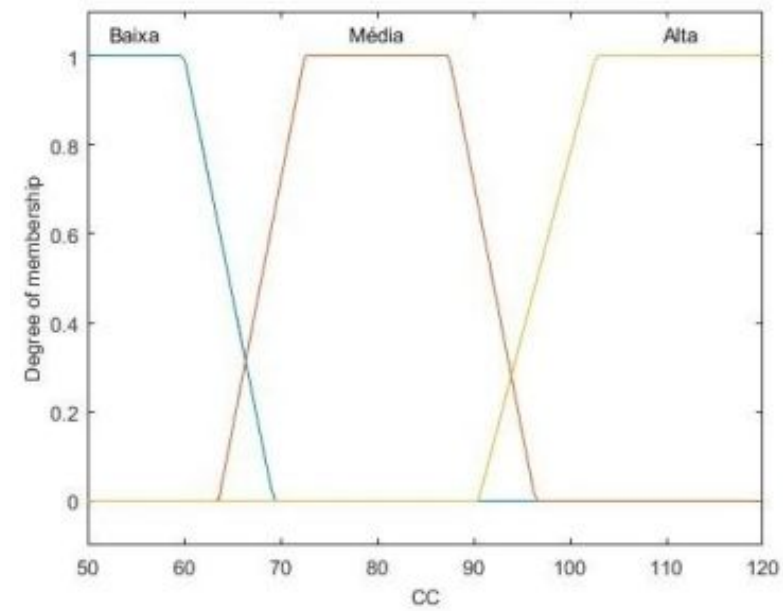

(c)

FIGURA 2. Funções de pertinência das variáveis de entrada. Fonte: (Elaborada pelos autores)

Destaca-se que os valores escolhidos para a classificação dessas variáveis foram aleatórios, justamente pelo fato de ser uma elaboração. A figura "2a" mostra que a massa corporal, os delimitadores utilizados foram, muito baixo, baixo, adequado, alto e muito alto, ambos os parâmetros foram colocados de maneira teórica, pois não se tem como predizer que homem ou mulher possui uma massa corporal dentro do padrão ou em excesso não sabendo suas variáveis antropométricas. A figura " $2 \mathrm{~b}$ " refere-se à altura, utilizou-se de maneira teórica os delimitadores baixa, média e alta. A figura "2c" aborda a circunferência da cintura, que também utilizou de maneira teórica os delimitadores como: baixa, média e alta. O modelo quando validado em trabalhos futuros, as funções de pertinência das variáveis de entrada serão com base nos dados coletados, e com parâmetros de medidas corporais existentes na literatura científica.

De acordo com o Quadro 4 para a variável de saída IC Fuzzy foram definidas risco baixo e risco alto com base no intervalo proposto por Valdez et al. (1993) e a sua função de pertinência na Figura 3 , os delimitadores para esta função de pertinência foram determinados como tipo trapezoidal. 
QUADRO 4. Definição das funções de pertinência da variável de saída IC Fuzzy.

\begin{tabular}{ccc}
\hline Conjuntos Fuzzy & Tipo & Delimitadores \\
\hline RB & Trapezoidal & {$[-1,411,31,4]$} \\
\hline RA & Trapezoidal & {$[1,31,41,72]$} \\
\hline
\end{tabular}

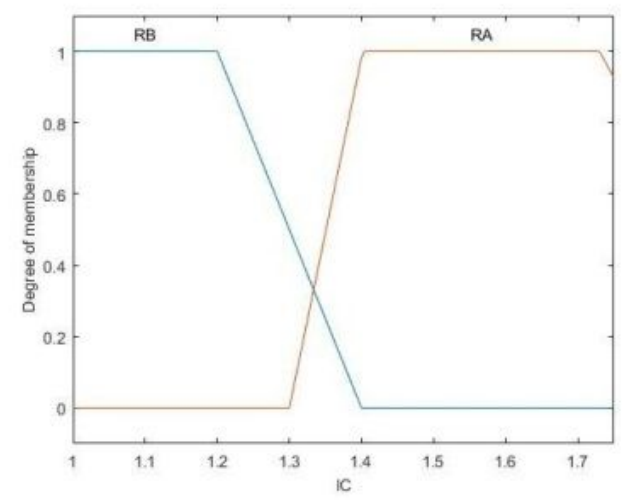

FIGURA 3. Funções de pertinência da variável de saída. Fonte: (Elaborada pelos autores)

Neste estudo elaborou-se um modelo computacional baseado em Lógica fuzzy, o mesmo não foi testado com dados reais, e sim aleatórios. No entanto, com a Lógica fuzzy foi possível elaborar um modelo, cujo o mesmo será testado e validado em trabalhos futuros a partir de dados reais, com intuito de auxiliar profissionais, pesquisadores e estudantes no cálculo matemático, bem como na avaliação através do intervalo estabelecido por Valdez et al. (1993).

Em Motamed et al. (2015) o IC foi indicado como uma variável forte para prever doenças cardiovasculares nos próximos 10 anos, comparado com o índice de volume abdominal. Outro estudo realizado com 319 adultos de $18-25$ anos, com o objetivo de determinar o efeito da obesidade central através do IC na idade pulmonar em adultos jovens, concluiu que idade pulmonar está significativamente elevada em adultos jovens obesos, centralmente em comparação com não obeso, além disso, o IMC mostrou-se não significativo e observou-se aumento do IC na presença de IMC normal. Poucos estudos indicam que as medidas de obesidade central são melhores discriminadores de morbidade em comparação com o IMC. Ao contrário do IMC, que não diferencia entre massa muscular, osso e gordura, o IC pode ser uma ferramenta útil para identificar indivíduos com obesidade central (SHENOY; JAGADAMBA, 2017).

David et al. (2018) realizaram um estudo com o objetivo de construir um modelo computacional fuzzy para estimar o número de internações de crianças até 10 anos devido a doenças respiratórias, utilizando dados de poluentes e fatores climáticos da cidade de São José do Rio Preto, Brasil. O modelo mostrou-se eficaz na predição do número de internações de crianças, sendo indicada sua utilização no âmbito hospitalar da região estudada, pois trata-se de um modelo de baixo custo e prático.

Silveira et al. (2018) realizaram um estudo com o intuito de elaborar um modelo matemático não testado, apenas elaborado de maneira teórica para analisar o risco de ataque cardíaco por meio da teoria de conjuntos fuzzy, levando em consideração fatores de risco que o paciente pode apresentar sendo: níveis de 
colesterol LDL considerado como um mal colesterol, e a concentração da proteína C-reativa presente no organismo que aumenta mediante a um processo inflamatório, e está relacionada com ataques cardíacos e a derrames cerebrais. Os resultados do estudo mostraram que a combinação entre os níveis de colesterol LDL e as concentrações da proteína C-reativa tem papel importante no que se refere a risco de infarto, observaram que o modelo conseguiu capturar as hipóteses contraditórias envolvidas no fenômeno, proporcionando respostas acessíveis com base na literatura.

O estudo de Coutinho et al. (2015) teve como objetivo construir um modelo computacional utilizando Lógica fuzzy para prever o tempo médio de internação de pacientes acometidos por doenças cardiovasculares causadas por poluentes do ar em uma cidade industrializada, o mesmo mostrou-se um modelo flexível e prático para a avalição em quaisquer localidades onde existam dados disponíveis de poluentes e condições climáticas, assim como em David et al. (2015) que ressaltaram a viabilidade do modelo bem como sua eficácia.

A Lógica fuzzy tem sido muito aplicada na área da saúde, pois auxilia de modo significativo no processo de tomada de decisão, entretanto é escassa a sua utilização em indicadores antropométricos, principalmente na aplicação para o cálculo do IC. Na prática clínica, esse índice pode ser obtido usando algumas medidas simples e rotineiras, porém exige conhecimentos matemáticos para a realização do cálculo, o que limita muitas vezes a utilização. Desse modo, a aplicação da Lógica fuzzy vem desempenhar uma avaliação do IC de maneira mais prática, visto que possibilita maior distinção entre os valores considerados chave para a propensão ou não a doenças cardiovasculares, por meio dos intervalos estabelecidos por Valdez et al. (1993).

\section{CONCLUSÃO}

Conclui-se que o uso da Lógica fuzzy pode contribuir na análise da obesidade abdominal auxiliando profissionais, pesquisadores e estudantes que queiram fazer 0 uso do IC em suas avaliações antropométricas. O modelo proposto vem como facilitador para os avaliadores já que mostra o resultado de forma prática e rápida sem cálculos a serem executados. No entanto, o modelo proposto será validado para seu uso com dados de medidas corporais de adultos em trabalhos futuros, apresentando ao modelo as bases de regras, para que assim possa avaliar o IC de cada indivíduo adulto, e comparar cada um individualmente. Este modelo é capaz de imitar parte do raciocínio humano, e foi baseado em Lógica fuzzy.

\section{AGRADECIMENTO}

Os autores agradecem ao Conselho Nacional de Desenvolvimento Científico e Tecnológico (CNPq) pela bolsa concedida de Iniciação Científica e a Bolsa de Produtividade em Pesquisa (Processo 313570/2017-5) que possibilitaram a realização do presente trabalho.

\section{REFERÊNCIAS}

AZEVEDO, P.S.; PAIVA, S.A.; ZORNOFF, L.A. Nutrition and cardiology: an interface not to be ignored. Arquivo Brasileiro de Cardiologia, v. 103 n. 2 p. 87-88, 2014. DOI: http://dx.doi.org/10.5935/abc.20140121

COUTINHO, K.M.V.; RIZOL, P.M.S.R.R.; NASCIMENTO, L.F.C., MEDEIROS, A.P.P. Fuzzy model approach for estimating time of hospitalization due to cardiovascular 
diseases. Ciência e Saúde Coletiva, v. 20, n.8, p. 2585-2590, 2015. DOI: http://dx.doi.org/10.1590/141381232015208.19472014

CREMASCO, C.P.; GABRIEL FILHO, L.R.A.; CATANEO, A. Methodology for determination of fuzzy controller pertinence functions for the energy evaluation of poultry industry companies. Energia na Agricultura, v.25, p. 21-39, 2010. DOI: http://dx.doi.org/10.17224/EnergAgric.2010v25n1p21-39

DAVID, G.S.; RIZOL, R.S.M.P.; NASCIMENTO, C.F.L. Modelos computacionais fuzzy para avaliar efeitos da poluição do ar em crianças. Revista Paulistana de

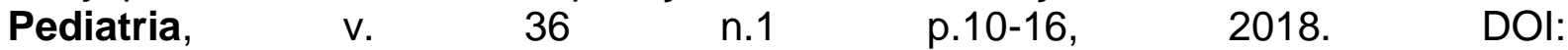
http://dx.doi.org/10.1590/1984-0462/;2018;36;1;00013

GABRIEL FILHO, L.R.A.; PIGATTO, G.A.S.; LOURENZANI, A.E.B.S. Fuzzy rulebased system for evaluation of uncertainty in cassava chain. Engenharia Agrícola, v.35, n.2, p.350-367, 2015. DOI: http://dx.doi.org/10.1590/1809-4430Eng.Agric.v35n2p350-367/2015

HAUN, D.R.; PITANGA, F.J.G.; LESSA, I. Razão cintura/estatura comparado a outros indicadores antropométricos de obesidade como preditor de risco coronariano elevado. Revista da Associação Médica Brasileira, v. 55 n.6 p. 705-711, 2009. DOI: http://dx.doi.org/10.1590/S0104-42302009000600015

MALTA, C.D.; BERNAL, I.T.R.; ANDRADE, A.C.S.S.; SILVA, A. M.M.; MELENDEZ, V.G. Prevalência e fatores associados com hipertensão arterial autorreferida em adultos brasileiros. Revista de Saúde Pública, v.51 n.1 p. 1-11, 2017. DOI: http://dx.doi.org/10.1590/s1518-8787.2017051000006

MARIEB, N.E.; WILHELM, B. P.; MALLATT, J. Anatomia Humana. 7 ed. São Paulo: Person Education do Brasil, 2014.890p.

MORAIS, R.S.; VIANA, C.C.A.; DALTRO.; S.C.F.A.; MENDONÇA, P.S. Risco cardiovascular e uso do índice de conicidade em pacientes submetidos ao transplante autólogo de células-tronco hematopoiéticas. Einstein, v. 18 n.2 p.1-5, 2018. DOI: http://dx.doi.org/10.1590/s1679-45082018ao4253

MOTAMED, N.; PERUMAL, D.; ZAMANI, F.; ASHRAFI, H.; HAGHJOO, M. et al. Conicity Index and Waist-to-Hip Ratio Are Superior Obesity Indices in Predicting 10Year Cardiovascular Risk Among Men and Women. Clinical Cardiology, v.38, n.9, p.527-534, 2015. DOI: http://dx.doi.org/10.1002/clc.22437

OLIVEIRA, M.A.M.; FAGUNDES, R.L.M.; MOREIRA, E.A.M.; TRINDADE, E.B.S.M.; CARVALHO, T. Relação de Indicadores Antropométricos com Fatores de Risco para Doença Cardiovascular. Arquivo Brasileiro de Cardiologia, v. 94, n. 4, p 478-485, 2010. DOI: http://dx.doi.org/10.1590/S0066-782X2010005000012

PEREIRA, D.F.; BIGHI, C.A.; GABRIEL FILHO, L.R.A.; CREMASCO, C.P.C. Sistema fuzzy para estimativa do bem-estar de matrizes pesadas. Engenharia Agrícola, v.28, n.4, p.624-633, 2008. DOI: http://dx.doi.org/10.1590/S010069162008000400002 
PERRONE-FILARDI, P.; PAOLILLO, S.; COSTANZO, P.; SAVARESE, G.; TRIMARCO, B. et al. The role of metabolic syndrome in heart failure. European Heart Journal, v. $36, \quad$ n.39, p. 2630-2634, $2015 . \quad$ DOI: http://dx.doi.org/10.1093/eurhearti/ehv350

PINTO, G. X.; PIAZENTIN, J. C.; BONINI NETO, A. Modelo de classificação automática para o índice de conicidade via redes neurais artificiais (RNAS). Colloquium Exactarum. v. 9 , n. 4, p. 48-55, 2018. Disponível em: http://journal.unoeste.br/index.php/ce/article/view/2268

PITANGA, F. J. G.; LESSA, I. Associação entre indicadores antropométricos de obesidade e risco coronariano em adultos na cidade de Salvador, Bahia, Brasil. Revista Brasileira de Epidemiologia, v.10, n.2, p.239-248, 2007. DOI: http://dx.doi.org/10.1590/S1415-790X2007000200011

PUTTI, F.F.; GABRIEL FILHO, L.R.A.; CREMASCO C.P.; BONINI NETO, A. BONINI, C.S.B. REIS, A.R. A Fuzzy mathematical model to estimate the effects of global warming on the vitality of Laelia purpurata orchids. Mathematical Biosciences, v.288, p.124-129, 2017. DOI: https://doi.org/10.1016/j.mbs.2017.03.005

PUTTI, F.F.; GABRIEL FILHO, L.R.A.; SILVA, A.O.; LUDWIG, R.; CREMASCO, C.P.C. Fuzzy logic to evaluate vitality of catasetum fimbiratum species (Orchidacea). Revista Irriga, v.19, n.3, p.405-413, 2014. DOI: http://dx.doi.org/10.15809/irriga.2014v19n3p405

SHENOY, U.; JAGADAMBA, A. Influence of central obesity assessed by conicity index on lung age in young adults. Journal of Clinical and Diagnostic Research, v.11, n. 4, p.CC09- CC012, 2017. DOI: http://dx.doi.org/10.7860 / JCDR / 2017 / 23428.9718

SILVEIRA, P.G.; PEREIRA, M.K.N.; GARCIA, O.R. Um estudo sobre o risco de ataque cardíaco via teoria de conjuntos fuzzy. Revista eletrônica paulista de matemática, v. $13 \quad$ n. 1 p. 99-117, 2018. DOI: http://dx.doi.org/10.21167/cqdvol13201823169664gpsnkmprog99117

TONSTAD S, HJERMANN I. A high risk score for coronary heart disease is associated with the metabolic syndrome in 40-year-old men and women. Journal Cardiovascular Risk factors, v.10, n.129-135, 2003. DOI: http://dx.doi.org/10.1177/174182670301000208

VALDEZ, R. A simple model-based index of ab - dominal adiposity. Journal of Clinical of Epidemiology v.44 n.9 p. 955-956, 1991. DOI: http://dx.doi.org/10.1016/0895-4356(91)90059-I

VALDEZ, R.; SEIDELL, J.C.; AHN, Y.I.; WEISS, K.M. A new index of abdominal adiposity as na indicator of risk for cardiovascular disease. A cross-population study. International Journal of Obesity and Related Metabolic disorders, v.17, n.2, p.77- 82,1993. Disponível em: <https://www.ncbi.nlm.nih.gov/pubmed/8384168>. 
WORLD HEALTH ORGANIZATION (WHO). Obesity and overweight, 2018. Disponível em: <https://www.who.int/news-room/fact-sheets/detail/obesity-andoverweight>. 